\title{
Self-assessment questions: The hepato-enteric immune axis in health and disease
}

\author{
Palak J Trivedi and David H Adams
}

1 The liver is able to generate a vigorous immune response to pathogenic organisms entering through the portal circulation while simultaneously being tolerogenic to harmless food-borne antigens. Which of the following statements regarding the hepatic immune response are true?

(a) A vigorous intrahepatic immune response depends on activation of T-cells by hepatic-resident antigenpresenting cells

(b) A vigorous intrahepatic immune response depends on activation of DCs by liver-resident T-cells

(c) Direct activation of T-cells within the liver by hepaticresident antigen-presenting cells usually results in tolerance

(d) Direct activation of DCs within the liver by hepatic-resident T-cells usually results in tolerance

(e) IL10 promotes the development of effector T-cells over $\mathrm{T}_{\text {reg }} \mathrm{s}$

2 Primary sclerosing cholangitis (PSC) is strongly associated with inflammatory bowel disease (IBD). Which of the following questions regarding PSC and IBD are correct?

(a) The majority of patients with IBD have PSC

(b) Patients with PSC and IBD are have a lower lifetime risk of developing colonic carcinoma than patients with IBD alone

(c) Colectomy for ulcerative colitis prevents the development of PSC

(d) Patients with PSC and IBD have a greater lifetime risk of developing colonic carcinoma than patients with IBD alone

(e) Crohn's disease is more common than ulcerative colitis in patients with PSC
3 Regarding enterohepatic lymphocyte homing, which of the following statements are false?

(a) CCL25 and $\alpha 4 \beta 7$ are constitutively expressed on the sinusoidal endothelium in normal human liver

(b) CCL25 and MAdCAM-1 are upregulated in the human liver in PSC

(c) Mucosal VAP-1 expression is upregulated in ulcerative colitis

(d) MAdCAM-1 is constitutively expressed on the lamina propria of the small bowel

(e) VAP-1 is constitutively expressed on the human liver sinusoidal endothelium

4 Which of the following statements is correct?

(a) Enzymatic action of MAdCAM-1 leads to the upregulation of VAP-1 expression

(b) Neutralising antibodies against MAdCAM-1 are efficacious in treating PSC

(c) MAdCAM-1 possesses amine oxidase activity and also functions as an adhesion molecule

(d) Patients who develop PSC after being diagnosed with IBD have a better outcome than those who are diagnosed with PSC before being diagnosed with IBD

(e) Colectomy for ulcerative colitis before liver transplant is more protective against PSC recurrence in the liver allograft than colectomy performed 10 years after transplant

Answers to these self-assessment questions can be found on page s92. 